\title{
Local density approach for modeling fluids with density-dependent interactions
}

\author{
N. G. Almarza, ${ }^{1}$ E. Lomba, ${ }^{1}$ G. Ruiz, ${ }^{2}$ and C. F. Tejero ${ }^{3}$ \\ ${ }^{1}$ Instituto de Química Física Rocasolano, CSIC, Serrano 119, E-28006 Madrid, Spain \\ ${ }^{2}$ Departamento de Matemática Aplicada y Estadística, EUIT Aeronáuticos, UPM, E-28040 Madrid, Spain \\ ${ }^{3}$ Facultad de CC Físicas, UCM, E-28040 Madrid, Spain
}

(Received 4 October 2002; published 12 February 2003)

\begin{abstract}
In a recent paper [Phys. Rev. Lett. 86, 2038 (2001)] a simple fluid with a particular density-dependent pair potential was shown to exhibit, together with the vapor-liquid transition, a liquid-liquid phase separation and it was evidenced that, in order to adequately define the correct boundaries of stability, a simulation procedure based on the use of local densities had to be devised. It was found that for certain thermodynamic states the potential drives the system toward a phase separation that is otherwise frustrated by the change in the interactions induced by density fluctuations. Therefore, when integral equations or global density simulations are used, the critical points estimated from the thermodynamics are not associated with divergent correlations and vice versa. Here, we will explore in depth this fluid and introduce a detailed account of the proposed local density simulation technique. The results presented bear general significance for density-dependent potentials, like those of liquid metals or charge-stabilized colloids.
\end{abstract}

DOI: 10.1103/PhysRevE.67.021202

PACS number(s): 61.20.Gy, 64.10.+h, 64.70.-p

\section{INTRODUCTION}

Density-dependent (or more generally state dependent) potentials play a key role in the theoretical modeling of complex fluids that would otherwise pose insurmountable difficulties given the typically large number of degrees of freedom involved [1]. Widely used treatments like the McMillan-Mayer theory of electrolytes where the effects of the solvent are integrated out and condensed into a state dependent dielectric constant fall into this class of simplifications, although perhaps one of the most representative cases corresponds to the effective ion-ion interaction in liquid metals [2] treated in the nearly free electron model. Here, the effects of the valence electron cloud appear in the effective ion-ion interaction mostly through the Fermi's wave number $k_{F}$ (a function of the electron density in turn). And moving from the atomic to the supramolecular level, we again encounter an example where state dependent potentials are of essential importance, namely, colloidal systems in which, according to the theory of Derjaguin-Landau-VerweyOverbeek [3,4], large charged colloidal particles immersed in electrolyte solutions experiment an effective screened Coulomb interaction. Here, the density of counterions (and hence the density of colloidal particles via the electroneutrality condition) enters the effective interaction through the screening constant and the effective valence. Density-dependent pair potentials have also been used to include implicitly the effects of three and higher-body interactions of real systems $[5,6]$. Clearly, the study of phase transitions in these systems is of primary importance. In this respect, recently it has been questioned whether simple one-component fluids can exhibit a liquid-liquid transition. A number of systems like water, $\mathrm{Si}$, $\mathrm{SiO}_{2}$, etc. (mostly tetrahedrally coordinated substances) seem to exhibit a liquid-liquid critical point in the supercooled region [7]. For a simple fluid model with a tunable density-dependent potential, Tejero and Baus [8] have shown that it is possible to generate a phase diagram within the van der Waals theory in which the liquid-liquid separation is well outside the metastable liquid-solid region. This potential model has the following form:

$$
V_{0}(r ; \rho)= \begin{cases}\infty & (r<\sigma) \\ -\epsilon\left(\frac{\sigma}{r}\right)^{n(\rho)} & (r \geqslant \sigma),\end{cases}
$$

where $\epsilon$ defines the amplitude of the potential (i.e., the temperature scale), $\sigma$ is the hard sphere diameter, $r$ is the interparticle distance, and $\rho$ is the density. The potential index $n(\rho)$ which determines the range of the interaction is given by

$$
n(\rho)=3+\frac{n(0)-3}{1-\frac{2}{3} \alpha \rho+\frac{1}{6} \alpha^{2} \rho^{2}} .
$$

As shown in Ref. [8] a wide variety of phase diagrams can be obtained by tuning the parameters $\alpha$ and $n(0)$ in Eq. (2).

In a recent paper [9], we have presented an integral equation, perturbation theory, and Monte Carlo(MC) simulation study for a slightly modified potential $V(r ; \rho)$. For practical purposes, $V_{0}(r ; \rho)$ was truncated and shifted, which does not qualitatively alter the features of the phase diagrams presented herein. The explicit form of the potential reads

$$
V(r ; \rho)=\left\{\begin{array}{l}
\infty \quad(r<\sigma) \\
V_{0}(r ; \rho)-V_{0}\left(r_{c} ; \rho\right) \quad\left(\sigma \leqslant r \leqslant r_{c}\right) \\
0 \quad\left(r>r_{c}\right),
\end{array}\right.
$$

where $r_{c}$ is the cut-off distance and $V_{0}(r ; \rho)$ is given by Eq. (1). This truncated and shifted potential also exhibits liquidliquid separation for certain combinations of $\alpha$ and $n(0)$. This liquid-liquid separation stems basically from the density dependence of the potential, and in this respect, it is closely connected to the phase separation found by Dijkstra and van 
Roij [10] for a purely repulsive density-dependent Yukawa potential. There is, however, a substantial difference between these two systems. The purely repulsive system will exhibit a transition stemming solely from the density dependence of the potential, for instance an isotherm computed in a canonical simulation might exhibit a van der Waals loop with little or none particle number dependence, contrary to the standard behavior in a phase separation process led by attractive forces. In contrast, the system studied in Refs. [8,9], due to the presence of attractions, might exhibit a tendency to phase separation which under certain circumstances is frustrated by the change of the interactions induced by the density fluctuations. This explains the large differences between the integral equation and the thermodynamic perturbation predictions. It was thus made clear in Ref. [9] that the treatment of potentials which exhibit a density dependence poses considerable methodological and conceptual problems (see Ref. [11]), and that a coherent simulation approach must account for the fact that the interactions change as a result of inhomogeneities in the sample. In this work, we intend to present an in-depth study of the potential model proposed in Ref. [8], both analyzing the difficulties encountered by the standard integral equation approaches and with special emphasis on the simulation procedure based on the use of local densities.

The rest of the paper is organized along the following lines. The following section is devoted to a brief presentation of the thermodynamics of density-dependent potentials. In Sec. III, we have collected the essentials of the thermodynamic perturbation treatment and the integral equation approach. In Sec. IV, we present some general remarks on a standard global density-dependent MC simulation. A full account of the simulation approach here proposed is presented in Sec. V. Finally, in Sec. VI the most significant results are collected and commented upon.

\section{THERMODYNAMICS AND PHASE SEPARATION IN SIMULATIONS WITH DENSITY-DEPENDENT POTENTIALS}

Let us first consider a system described by the potential model, Eqs. (1) and (2), with a fixed potential index $n_{0}$ $\equiv n\left(\rho_{0}\right)$. If the corresponding Helmholtz free energy is denoted by $F_{0}=F_{0}\left(\beta, V, N \mid n_{0}\right)$, where $\beta=1 / k_{B} T$, with $k_{B}$ Boltzmann's constant and $T$ the absolute temperature, $N$ is the number of particles, $V$ is the volume, and the implicit dependence on $n_{0}$ has also been expressed, we have

$$
d\left(\beta F_{0}\right)=U_{0} d \beta-\beta p_{0} d V+\beta \mu_{0} d N,
$$

where $U_{0}$ is the internal energy, $p_{0}$ stands for the pressure, and $\mu_{0}$ is the chemical potential.

Focusing now on a system described by the potential model, Eqs. (1) and (2) in which the potential index $n(\rho)$ is a function of the density, the differential form of the corresponding Helmholtz free energy $F=F(\beta, V, N \mid n(\rho))$ is

$$
d(\beta F)=U d \beta-\beta p d V+\beta \mu d N
$$

where $U, p$, and $\mu$ denote the internal energy, the pressure, and the chemical potential, respectively.
Note that although $F\left(\beta, V, N \mid n\left(\rho_{0}\right)\right)=F_{0}\left(\beta, V, N \mid n_{0}\right)$, the free-energy derivatives for this thermodynamic state do not, in general, coincide. Indeed, the implicit dependence on $N$ and $V$ through the potential index $n(\rho)$ leads to

$$
p=-\left(\frac{\partial F}{\partial V}\right)_{\beta, N, n(\rho)}-\left(\frac{\partial F}{\partial n(\rho)}\right)_{\beta, V, N}\left(\frac{\partial n(\rho)}{\partial V}\right)_{N}
$$

and

$$
\mu=\left(\frac{\partial F}{\partial N}\right)_{\beta, V, n(\rho)}+\left(\frac{\partial F}{\partial n(\rho)}\right)_{\beta, V, N}\left(\frac{\partial n(\rho)}{\partial N}\right)_{V} .
$$

In the thermodynamic limit, the pressures ( $p$ and $p_{0}$ ) and the chemical potentials $\left(\mu\right.$ and $\left.\mu_{0}\right)$ are functions of $\beta$ and $\rho$, and Eqs. (6) and (7) reduce to

$p(\beta, \rho \mid n(\rho))=p_{0}\left(\beta, \rho \mid n_{0}\right)+l(\beta, \rho \mid n(\rho)) \rho^{2} n^{\prime}(\rho) \quad\left(\rho=\rho_{0}\right)$

and

$$
\begin{aligned}
& \mu(\beta, \rho \mid n(\rho)) \\
& \quad=\mu_{0}\left(\beta, \rho \mid n_{0}\right)+l(\beta, \rho \mid n(\rho)) \rho n^{\prime}(\rho) \quad\left(\rho=\rho_{0}\right),
\end{aligned}
$$

where $n^{\prime}(\rho)$ denotes the derivative of the potential index with respect to the density and $l(\beta, \rho \mid n(\rho))$ is the thermodynamic limit of

$$
l(\beta, V, N \mid n(\rho)) \equiv \frac{1}{N}\left(\frac{\partial F}{\partial n(\rho)}\right)_{\beta, V, N} .
$$

Let us assume that we perform a simulation in the canonical $N V T$ ensemble and that at the thermodynamic state $\left(\beta, \rho_{0}\right)$, we have

$$
\left(\frac{\partial p(\beta, \rho \mid n(\rho))}{\partial \rho}\right)_{\rho=\rho_{0}} \leqslant 0,\left(\frac{\partial p_{0}\left(\beta, \rho \mid n_{0}\right)}{\partial \rho}\right)_{\rho=\rho_{0}}>0,
$$

In such a case, the first condition implies that this thermodynamic state is within a van der Waals loop in the $p-\rho$ plane but, due to the second condition, at this state there will be no signature of segregation. For instance, the loop will show little dependence on the number of particles, the size dependence of the fluid-fluid equilibrium binodal close to the critical point will be negligible, and the critical exponents will not be affected by long range fluctuations. In this situation, one can perform the simulation without major problems also in the $N p T$ or grand canonical ensembles [12].

On the other hand, it is possible to find that at the thermodynamic state $\left(\beta, \rho_{0}\right)$

$$
\left(\frac{\partial p(\beta, \rho \mid n(\rho))}{\partial \rho}\right)_{\rho=\rho_{0}}>0,\left(\frac{\partial p_{0}\left(\beta, \rho \mid n_{0}\right)}{\partial \rho}\right)_{\rho=\rho_{0}} \leqslant 0,
$$

i.e., the system should be homogeneous according to the thermodynamic stability criteria, but in practice the simulated system is not. If such is the case, a system simulated on 
a NVT ensemble will show some tendency to separate into different phases, which reflects in the strong system size dependence of the $p_{0}$ values.

The source of this contradiction lies in a flaw of the standard simulation procedure when dealing with densitydependent potentials. In order to determine the interactions, in practice, one uses a potential index $n(\rho)$ that is determined by the average value of the density $\rho$ (hereafter, referred to as the "global" density) in the whole system, irrespective of the presence of inhomogeneities. Thus, if the system separates into two phases of $N_{\text {I }}$ particles in a volume $V_{\mathrm{I}}$ and $N_{\text {II }}$ particles in a volume $V_{\text {II }}$, with $\rho_{\mathrm{I}}=N_{\mathrm{I}} / V_{\mathrm{I}}$ and $\rho_{\mathrm{II}}=N_{\mathrm{II}} / V_{\mathrm{II}}$, the standard approach implies that the interactions in each phase are identical and controlled by a potential index $n(\rho)$. However, if each phase is considered as a separate subsystem we will have two different potential indices $n_{\mathrm{I}}=n\left(\rho_{\mathrm{I}}\right)$ and $n_{\mathrm{II}}=n\left(\rho_{\mathrm{II}}\right)$ and hence, if we divide the system into two parts it is straightforward to conclude that the Helmholtz free energy will no longer properly behave as an extensive variable. Indeed, the free energy $F_{N V T}(\beta, V, N \mid n(\rho))$ that controls the evolution of the global system is

$$
\begin{aligned}
F_{N V T}(\beta, N, V \mid n(\rho))= & F_{0}\left(\beta, N_{I}, V_{I} \mid n(\rho)\right) \\
& +F_{0}\left(\beta, N_{I I}, V_{I I} \mid n(\rho)\right),
\end{aligned}
$$

whereas the proper free energy associated with the physical situation under scrutiny should be

$$
F(\beta, N, V \mid n(\rho))=F_{0}\left(\beta, N_{\mathrm{I}}, V_{\mathrm{I}} \mid n_{\mathrm{I}}\right)+F_{0}\left(\beta, N_{\mathrm{II}}, V_{\mathrm{II}} \mid n_{\mathrm{II}}\right) .
$$

In $N p T$ simulations of one-component systems with densityindependent pair potentials, the simultaneous presence of two phases in the simulation box is hindered by the surface tension. For a large system with density-dependent interactions, we can find phase coexistence at the transition pressure if the free-energy penalty due to the presence of interfaces is compensated. It will then be possible again to encounter situations where the Gibbs free energy is no longer an extensive property. This would be the case when the density dependence of the potential index $n(\rho)$ leads to less attractive interactions as density increases. If the system separates in two phases inside the simulation box, the phase with higher density will have a lower Gibbs free energy than a corresponding homogeneous high density phase, if the interactions are evaluated using the global average density. This reduction of Gibbs free energy can compensate the increase of free energy of the low density (where the pair potential will be less attractive than the one corresponding to a homogeneous phase of the same density) and the interfacial free energy due to the presence of two phases at equilibrium.

Similar problems will appear in the grand canonical ensemble, where in principle only one phase is expected to appear in the simulation box in the thermodynamic limit. This is because the excess of free energy due to the formation of interfaces can be overcompensated by the use on a potential index, $n(\rho)$ equal in both phases.

Use of the Gibbs ensemble [13] is also not free of problems. For large systems, we can find a situation similar to the one described for the canonical case. The phase separation can occur in such a way that both boxes contain the two phases and similar global densities, instead of having one phase in each box.

\section{ESSENTIALS OF THE INTEGRAL EQUATION AND THERMODYNAMIC PERTURBATION APPROACHES}

In this section, we present a brief account of two common theoretical approaches to describe the thermodynamics of a fluid with the potential model (3), namely, Ornstein-Zerniketype integral equations and high temperature thermodynamic perturbation theory (TPT).

Different integral equation theories of the radial distribution function $g(r ; \rho)$ are defined in terms of the so-called bridge function $b(r ; \rho)$, which involves high order particle correlations so that an approximation for $g(r ; \rho)$ can be obtained. We will focus our attention on two approximation schemes, the hypernetted chain (HNC) and the reference HNC (RHNC). As it is well known, these approximation schemes consist of the Ornstein-Zernike relation

$$
h(r ; \rho)=c(r ; \rho)+\rho \int c\left(\left|\mathbf{r}-\mathbf{r}^{\prime}\right| ; \rho\right) h\left(\mathbf{r}^{\prime} ; \rho\right) d \mathbf{r},
$$

where $h(r ; \rho)=g(r ; \rho)-1$, with $c(r ; \rho)$ being the direct correlation function, together with a closure of the form

$$
g(r ; \rho)=\exp [-\beta V(r ; \rho)+h(r ; \rho)-c(r ; \rho)+b(r ; \rho)],
$$

where $V(r ; \rho)$ is the interparticle potential. The bridge function $b(r ; \rho)$ can simply be neglected, leading to the HNC closure, or approximated by the bridge function of a hard sphere (HS) fluid yielding the RHNC closure. In the spirit of Lado, Foiles, and Ashcroft optimized approach [14], we have also set the hard sphere diameter so as to minimize the free energy. It is well known that the HNC thermodynamics is fully self-consistent, aside from the isothermal compressibility calculated from the fluctuation theorem, which cannot be derived from the HNC functional [15]. Hence, the pressure calculated via the virial equation is consistent with that obtained from the density derivative of the free energy and with the one derived from the energy route. These consistency properties hold approximately in the RHNC, but additionally, in the case of density-independent potentials the discrepancies between the virial and the compressibility equations are minimized. For density-dependent potentials, however, the latter consistency property is ruled out. This is due to the fact that the usual form of the compressibility equation,

$$
1+\rho \int h(\mathbf{r} ; \rho) d \mathbf{r}=\rho k_{B} T \kappa_{T},
$$

where $\kappa_{T}$ is the isothermal compressibility coefficient, no longer holds $[16,17]$. As a consequence, the thermodynamic critical points are not associated with diverging correlations, and, therefore, a no solution curve of an integral equation is not necessarily the signature of a phase transition any more, and vice versa. Thus, for the potential model (3), the pressure 


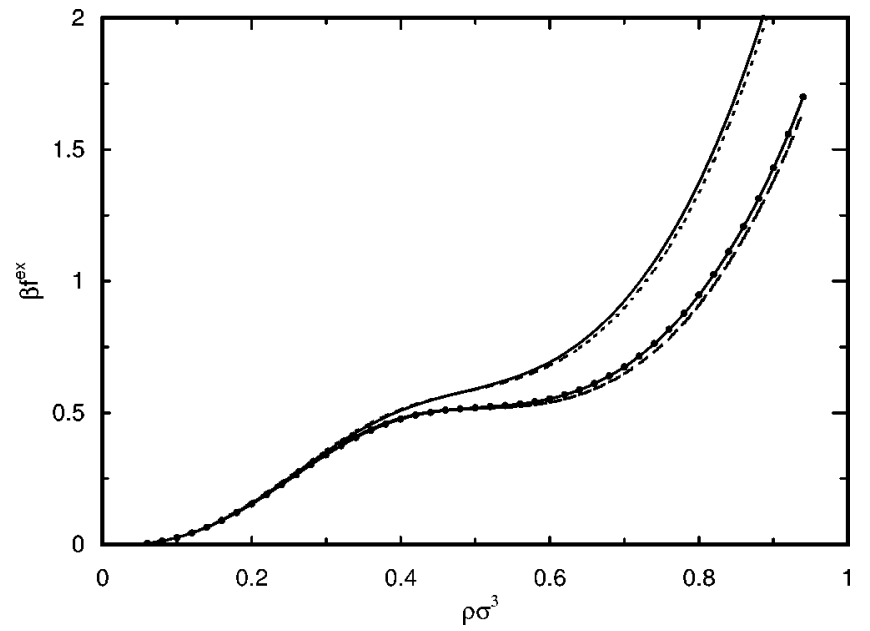

FIG. 1. Reduced excess free energy $\beta f_{e x}$ versus the reduced density, as obtained from the HNC (solid line) and RHNC (solid line with circles) functionals, and from thermodynamic integration of the virial pressure for the HNC (dotted line) and RHNC (dashed line) for $\alpha=12 v_{H S}, n(0)=3.5, r_{c}=5.1 \sigma$, and $t=k_{B} T / \epsilon=3$.

exhibits a well defined van der Waals loop, a feature that is typically absent for HNC-like approximations for state independent potentials, i.e., in these cases, both (HNC and RHNC) equations break down before the pressure or the chemical potential can develop a van der Waals loop. Moreover, we observe in Fig. 1, the high degree of consistency between the free-energy functional [15] and the free energy obtained from thermodynamic integration of the virial pressure. On the other hand, when comparing with MC results, the RHNC as expected is somewhat superior to the HNC approximation. Consequently, in what follows, we will just concentrate on the RHNC results.

Satisfactory estimates of the thermodynamic quantities can also be obtained in the context of TPT. As usual, we split the pair potential as

$$
V(r ; \rho \mid \lambda)=V^{H S}(r)+\lambda V^{A}(r ; \rho),
$$

using the HS fluid as the reference system and the attractive (density-dependent) potential as the perturbation. The parameter $\lambda(0 \leqslant \lambda \leqslant 1)$ stands for the coupling term which gradually "switches on" the perturbation.
The excess free energy per particle can be written as

$$
f_{e x}=f_{e x}^{H S}+\frac{1}{N} \int_{0}^{1}\left\langle H_{N}^{A}\right\rangle_{\lambda} d \lambda,
$$

where $\left\langle H_{N}^{A}\right\rangle_{\lambda}$ is the canonical ensemble average of the total perturbation energy, and $f_{e x}^{H S}$ is the excess free energy per particle of the HS fluid.

Using a high temperature expansion truncated at first order [18], it can be shown that $f_{e x}$ can be approximated by

$$
f_{e x}=f_{e x}^{H S}+\frac{1}{2} \rho \int g_{H S}(r ; \rho) V^{A}(r ; \rho) d \mathbf{r},
$$

where $g_{H S}(r ; \rho)$ is the radial distribution function of the HS reference system.

Comparing the first-order TPT results with the RHNC estimates (see Table I), we conclude that we can confidently rely on the TPT predictions to qualitatively describe the phase behavior of the potential model (3). The TPT is not plagued with the convergence difficulties present in the RHNC, which to make matters worse in the present instance are not correlated with the phase transition.

Finally, in order to obtain the coexistence curves, one simply has to solve the two-phase coexistence conditions

$$
p\left(\rho_{\mathrm{I}}, T\right)=p\left(\rho_{\mathrm{II}}, T\right)
$$

and

$$
\mu\left(\rho_{\mathrm{I}}, T\right)=\mu\left(\rho_{\mathrm{II}}, T\right),
$$

where the pressure $p$ and the chemical potential $\mu$ can be derived from the free energy per particle $f=k_{B} T\left[\ln \left(\rho \Lambda^{3}\right)\right.$ $-1]+f_{\text {ex }}$, with $\Lambda$ the thermal de Broglie wavelength, using the thermodynamic relations

$$
p=\rho^{2} \partial f / \partial \rho, \quad \mu=\partial(\rho f) / \partial \rho .
$$

\section{THE SIMULATION PROCEDURE USING GLOBAL DENSITIES}

As pointed out, we first consider global densities on the MC simulations for the potential model (3). In systems with a small number of particles and large density, it may happen

TABLE I. Reduced excess free energy per particle $\beta f^{e x}$, internal energy per particle $\beta u$, and pressure $p^{*}=\beta p \sigma^{3}$ from first-order TPT and RHNC for reduced temperature $t=k_{B} T / \epsilon=3.0$ at different reduced densities $\rho^{*}=\rho \sigma^{3}$.

\begin{tabular}{lcccccc}
\hline \hline$\rho^{*}$ & $\beta f_{T P T}^{e x}$ & $\beta f_{R H N C}^{e x}$ & $\beta u_{T P T}$ & $\beta u_{R H N C}$ & $\left(p^{*}\right)_{T P T}$ & $\left(p^{*}\right)_{R H N C}$ \\
\hline 0.1 & 0.032 & 0.026 & -0.192 & -0.204 & 0.108 & 0.108 \\
0.2 & 0.160 & 0.153 & -0.321 & -0.337 & 0.269 & 0.270 \\
0.3 & 0.348 & 0.340 & -0.433 & -0.448 & 0.462 & 0.463 \\
0.4 & 0.484 & 0.476 & -0.647 & -0.661 & 0.535 & 0.529 \\
0.5 & 0.528 & 0.519 & -1.02 & -1.03 & 0.552 & 0.527 \\
0.6 & 0.565 & 0.553 & -1.48 & -1.49 & 0.847 & 0.789 \\
0.7 & 0.692 & 0.675 & -1.97 & -1.98 & 1.66 & 1.56 \\
0.8 & 0.975 & 0.948 & -2.45 & -2.45 & 3.23 & 3.08 \\
\hline \hline
\end{tabular}


that $L<2 r_{c}$, where $L$ is the length of the side of the simulation box. In these cases we have explicitly calculated the pair interactions for $r<L / 2$, and a mean-field correction [19] has been added to the energy to take into account the interactions in the range $\left[L / 2, r_{c}\right]$. The chemical potential can be evaluated using the insertion method [19].

$$
\beta \mu \simeq \ln \left(\rho \Lambda^{3}\right)-\ln \left\langle e^{-\beta \Delta U}\right\rangle_{N V T},
$$

where $\Delta U$ accounts for the change of energy of the system when introducing a test particle at a random position in the system. The density dependence of the potential index $n(\rho)$ has to be taken into consideration, so that $\Delta U$ has two contributions: the change due to the new value of the potential index for the system with $N+1$ particles due to the increase of the density, and the change due to the interactions of the test particle with the remaining $N$ particles in the system.

For a fixed temperature, we can write

$$
\beta \mu(\rho)=\beta \mu^{0}+\ln z,
$$

where $z$ is the fugacity of the fluid and $\mu^{0}$ is a reference chemical potential (which only depends on the temperature). The usual choice for $\beta \mu^{0}$ is

$$
\beta \mu^{0}=\ln \left(\Lambda^{3} / \sigma^{3}\right) .
$$

The knowledge of $z$ for different values of $\rho$ defines the equation of state of the system. Expanding $\ln z$ as a series in the density

$$
\ln z=\ln \left(\rho \sigma^{3}\right)+\sum_{k=1} a_{k}\left(\rho \sigma^{3}\right)^{k}
$$

where the coefficients $a_{k}$ are estimated by fitting the simulation data. Using elementary thermodynamics one can express the coefficients of the virial expansion of the pressure in terms of $a_{k}$, leading to

$$
\beta p \sigma^{3}=\rho \sigma^{3}+\sum_{k=1} \frac{k}{k+1} a_{k}\left(\rho \sigma^{3}\right)^{k+1} .
$$

The determination of the phase equilibrium (18) and (19) is straightforward using Eqs. (24) and (25).

\section{THE SIMULATION PROCEDURE WITH LOCAL DENSITY CONSIDERATIONS}

It was shown in Sec. II that in certain cases the use of a global density in the definition of the pair potential leads to thermodynamic inconsistencies. In this section we will show how to circumvent this problem introducing a local density approach.

\section{A. Definition of the local density}

Consider a HS fluid with global density $\rho$. A local density $\rho_{L}$ can be defined by
TABLE II. Local densities from simulation and theory. See the text for details.

\begin{tabular}{ccccc}
\hline \hline$q$ & $\lambda$ & $\pi \rho \sigma^{3} / 6$ & $\left(\rho_{L} / \rho\right)_{\operatorname{sim}}$ & $\left(\rho_{L} / \rho\right)_{C S}$ \\
\hline 4 & 3.0 & 0.475 & 1.050 & 1.053 \\
6 & 3.0 & 0.475 & 1.047 & 1.044 \\
4 & 3.5 & 0.475 & 1.035 & 1.034 \\
4 & 4.0 & 0.475 & 1.024 & 1.023 \\
6 & 4.0 & 0.475 & 1.019 & 1.019 \\
6 & 3.0 & 0.300 & 1.039 & 1.037 \\
4 & 3.5 & 0.300 & 1.028 & 1.029 \\
6 & 4.0 & 0.300 & 1.016 & 1.016 \\
\hline \hline
\end{tabular}

$$
\rho_{L}=\rho \frac{\int g_{H S}(r ; \rho) w(r) d \mathbf{r}}{\int w(r) d \mathbf{r}}=\rho+\rho \frac{\int h_{H S}(r ; \rho) w(r) d \mathbf{r}}{\int w(r) d \mathbf{r}},
$$

where $h_{H S}(r ; \rho)=g_{H S}(r ; \rho)-1$ is the total correlation function of the HS fluid, $w(r)$ is a weight function of the form

$$
w(r)=\left[1-\left(\frac{r / \sigma-1}{\lambda-1}\right)^{q}\right]^{2} \quad(\sigma \leqslant r \leqslant \lambda \sigma),
$$

and zero otherwise, and $q$ is a positive integer, and $\lambda>1$. For certain values of $q$ and $\lambda, w(r) \simeq 1$ for short distances, and decays rapidly to zero at $r=\sigma \lambda . g_{H S}(r ; \rho)$ deviates significantly from unity precisely within this short range. Therefore, assuming that most of the contributions to the integral stem from the short range region (where $w \simeq 1$ ), we can make the following approximation:

$$
\rho \int h_{H S}(r ; \rho) w(r) d \mathbf{r} \simeq \rho \int h_{H S}(r ; \rho) d \mathbf{r}=\rho k_{B} T \kappa_{T}^{H S}-1,
$$

where $\kappa_{T}^{H S}$ is the isothermal compressibility coefficient of the HS fluid. Therefore, the local density can be estimated as

$$
\rho_{L}=\rho+\frac{\rho k_{B} T \kappa_{T}^{H S}-1}{\int w(r) d \mathbf{r}} .
$$

The accuracy of Eq. (29) can be assessed evaluating the local density $\rho_{L}$ by computer simulation for a HS fluid and comparing the results with those estimated using Eq. (29) and the Carnahan-Starling(CS) equation of state [18]. The parameters $q$ and $\lambda$ in Eq. (27) are tuned, so that the value of $\left(\rho_{L} / \rho\right)_{\text {sim }}$ and $\left(\rho_{L} / \rho\right)_{C S}$ are reasonably close. This led us to choose $q=6$ and $\lambda=4$ (see Table II).

In computer simulation the local density around a particle $i$ for a given configuration of the system can be evaluated from 


$$
\rho_{L, i}=\frac{\sum_{j \neq i} w\left(r_{i j}\right)}{\int w(r) d \mathbf{r}},
$$

where $r_{i j}$ is the distance between particles $i$ and $j$. Our goal is to design a simulation procedure for pair potentials that depend explicitly on the local densities. For homogeneous systems the method must render results essentially equivalent to those obtained for potentials which depend on the global density. The local densities evaluated through Eq. (29) are expected to be larger than the global density of a corresponding homogeneous system [due to the structure of $g(r ; \rho)$ at short distances]. Therefore, we should either increase the value of $\lambda$ to reduce such differences or to construct a device that relates a local density around a given particle, $\rho_{L, i}$ with an equivalent global density $\rho_{i}$. The second alternative has been chosen because of its lower computational cost. An accurate prescription for $\rho_{i}$ given the value of $\rho_{L, i}$ can be formulated using the scheme developed above. The differences between $\rho_{L}$ and $\rho$ for homogeneous systems are more significant at high densities. Moreover, the density dependence of $\rho_{L}$ must be monotonic in order to evaluate $\rho_{i}$ as a function of $\rho_{L, i}$. Such a condition is fulfilled using the CS equation of state. The determination of $\rho_{i}$ in terms of $\rho_{L, i}$ can be carried out by an iterative procedure. The $k$ th iteration estimate reads

$$
\rho_{i}^{(k)}=\rho_{L, i} \frac{\rho_{i}^{(k-1)}}{\rho_{L}\left(\rho_{i}^{(k-1)}\right)} .
$$

As initial solution one can use $\rho_{i}^{(0)}=\rho_{L, i}$.

\section{B. The potential energy in the local density model}

Since the local density varies from particle to particle, two particles $i$ and $j$ have, in general, different potential indices associated with their respective local densities. The pair interaction can then be defined as the average of the energies calculated with both potential indices, $n\left(\rho_{i}\right)$ and $n\left(\rho_{j}\right)$. Nonetheless, such a computational scheme is clearly impractical. If a particle $i$ changes its position in a trial move, the particles around it will undergo a change in their potential index and many pair interactions should be recalculated to evaluate the acceptance criteria. In order to develop a more efficient alternative each potential index $n_{i}$ has been treated as an "internal" coordinate of the particle $i$. These coordinates are relatively free to change from their "central values" $n\left(\rho_{i}\right)$. The condition of similarity between local and global density results (correspondence principle) for homogeneous systems implies that the differences $n_{i}-n\left(\rho_{i}\right)$ must remain small. This can be enforced introducing a new energy term in the Hamiltonian. The potential energy $U$ can then be written as

$$
U=U_{H S}+U_{\text {inter }}+U_{\text {intra }},
$$

where $U_{H S}$ corresponds to the hard sphere interactions and the intermolecular contribution $U_{\text {inter }}$ stands for the sum of pair interactions, similar to those present in the global density simulations but taking into account the particular values of the potential indices $n_{i}$ for the pair of particles involved

$$
U_{\text {inter }}=\frac{1}{2} \sum_{i} \sum_{j \neq i} V\left(r_{i j} ; n_{i}\right) \quad\left(r_{i j} \geqslant \sigma\right) .
$$

The "intramolecular" contribution $U_{\text {intra }}$ is chosen to be

$$
\beta U_{\text {intra }}\left(n_{i}\right)=\frac{K}{2} \sum_{i=1}^{N}\left[n_{i}-n\left(\rho_{i}\right)\right]^{2} .
$$

In this contribution, we include the many body interactions underlying the local density approach through the dependence of $\rho_{i}$ on the positions of the other particles. The value of $K$ must be large enough to avoid large fluctuations of $n_{i}$ around $n\left(\rho_{i}\right)$, while keeping an adequate acceptance rate in the MC simulation.

\section{Simulation algorithm}

Two kind of trial moves are performed in the simulation procedure: translations of a particle $i$ and changes of its internal coordinate. The first type of move is performed by standard procedures [19]. In order to apply the acceptance criteria, first the possibility of hard sphere overlaps is checked by a link-list procedure [19]. If the trial configuration is not forbidden by hard core overlaps, the change in the potential energy has to be evaluated. The calculation of $\Delta U_{\text {inter }}$ must incorporate two contributions with different potential indices for each pair interaction. The change in $U_{\text {intra }}$ is due to the variation of the local densities of the displaced particle and of the remaining particles in the system. The change in local densities is straightforward since the distances $r_{i j}$ are explicitly computed to calculate $\Delta U_{\text {inter }}$. For $j \neq i$,

$$
\rho_{L, j}^{n e w}=\rho_{L, j}+\frac{\left[w\left(r_{i j}^{n e w}\right)-w\left(r_{i j}\right)\right]}{\int w(r) d \mathbf{r}} .
$$

Once one has determined the new values of the local densities it is possible to calculate the corresponding equivalent global density $\rho_{j}^{\text {new }}$ to finally compute $\Delta\left(\beta U_{\text {intra }}\right)$. The acceptance criterion used for these moves is the standard one due to Metropolis [19].

In the second type of MC steps (sampling one internal coordinate, $n_{i}$ ) local densities do not change. The probability of a potential index $n_{i}$ (keeping constant the remaining coordinates of the system) is

$$
P\left(n_{i}\right) \propto \exp \left[-\beta W\left(n_{i}\right)\right],
$$

where

$$
\beta W\left(n_{i}\right)=\frac{K}{2}\left[n_{i}-n\left(\rho_{i}\right)\right]^{2}+\frac{\beta}{2} \sum_{j \neq i} V\left(r_{i j} ; n_{i}\right) \quad\left(r_{i j} \geqslant \sigma\right) .
$$


A function $W_{0}$ is defined from the Taylor expansion of $W\left(n_{i}\right)$ around $n\left(\rho_{i}\right)$ truncated to second order on $n_{i}$. The result can be expressed as

$$
\beta W_{0}\left(n_{i}\right)=a_{0}+\frac{K_{0}}{2}\left(n_{i}-n_{\text {min }}\right)^{2},
$$

where $n_{\min }$ is the value of the index that minimizes $\beta W_{0}$. Within the above conditions (large value of $K$ ), we have $K_{0} \simeq K$ and $n_{\text {min }} \simeq n\left(\rho_{i}\right)$. The trial value of $n_{i}, n^{\text {new }}$ is chosen with probability $P_{0}$

$$
P_{0}\left(n^{n e w}\right) \propto \exp \left[-\beta W_{0}\left(n^{n e w}\right)\right] .
$$

Detailed balance is fulfilled [19] when the acceptance probability $A$ of a change from $n_{i}=n^{\text {old }}$ to $n_{i}=n^{\text {new }}$ is given by

$$
A\left(n^{\text {new }} \mid n^{\text {old }}\right)=\max \left[1, \frac{P\left(n^{\text {new }}\right)}{P\left(n^{\text {old }}\right)} \frac{P_{0}\left(n^{\text {old }}\right)}{P_{0}\left(n^{\text {new }}\right)}\right] .
$$

For $K=10^{4}$, nearly $100 \%$ of the moves were accepted.

\section{Estimation of the chemical potential}

In order to evaluate the chemical potential, a range of acceptable values of the potential index has been defined, namely,

$$
n \in\left[n_{\min }, n_{\max }\right], \quad n_{\min }>3 .
$$

In principle, we can estimate the chemical potential by a method similar to the one used for global density simulations. Now one should also pick a value for the potential index of the test particle. A uniform sampling over its definition range is likely to give poor statistics on the estimation of $\mu$. This can be bypassed using procedures similar to those we have used in sampling internal coordinates. However, we found that such a strategy alone does not lead to a really efficient method. In the determination of the chemical potential of systems with a hard core we can consider two sources of uncertainty. First, it is required to have a sufficient number of successful insertions (i.e., without hard core overlaps) and second the fluctuation of the quantity to be averaged over successful insertions must not be very large. Special procedures are available [19] to cope with the first problem. In our case, given the low computational cost of checking hard sphere overlaps and that the densities of the systems are not too high in most of the cases, a direct insertion method is adequate. In order to solve the second problem, we analyzed the sources of large fluctuations in the insertion energy $\Delta U$ in successful insertions. Aside from the intramolecular energy of the test particle, the main source of fluctuation was found to be the changes on intramolecular energies of particles whose local densities are affected by the insertion. To avoid these effects, we employ a procedure in which, in the trial insertion, we keep constant the differences $\xi_{i}=n_{i}$ $-n\left(\rho_{i}\right)$. This scheme implies a simple change on the choice of internal coordinates. The chemical potential $\mu$ can be evaluated as

$$
e^{-\beta \mu}=\frac{\Delta n}{\rho \Lambda^{3} \Lambda_{n}}\left\langle e^{-\beta \Delta U}\right\rangle
$$

where $\Delta n \equiv n_{\max }-n_{\min }$ and $\Lambda_{n}$ takes into account the kinetic energy contribution of the internal coordinates to the partition function. Let $X_{0}$ be the fraction of successful insertions, then

$$
\left\langle e^{-\beta \Delta U}\right\rangle=X_{0} X_{s}
$$

where $X_{s}$ is the average of $\exp [-\beta \Delta U]$ over the successful insertions. Let $N_{s}$ be the total number of successful insertions, then $X_{s}$ can be computed from

$$
X_{s}=\frac{1}{N_{s} \Delta n} \sum_{k=1}^{N_{s}} \int_{n_{\min }}^{n_{\max }} d n \exp \left[-\beta \Delta U\left(\mathbf{r}_{k}, n\right)\right],
$$

where $\mathbf{r}_{k}$ denotes the position of the test particle in the successful insertion trial $k$. An uniform sampling on $n$ is expected to be inefficient. To cope with this, we replace $n$ with a new variable $\alpha$,

$$
\alpha=\operatorname{erf}\left[\sqrt{\frac{K_{\alpha}}{2}}\left(n-n_{\alpha}\right)\right]
$$

where erf is the error function, and the parameters $K_{\alpha}$ and $n_{\alpha}$ will be specified later. The change of variable leads to

$$
X_{s}=\frac{1}{N_{s} \Delta n} \sum_{k=1}^{N_{s}} \sqrt{\frac{\pi}{2 K_{\alpha}}} \int_{\alpha_{\text {min }}}^{\alpha_{\max }} \exp \left[-\beta \Delta U_{\text {ins }}\left(\mathbf{r}_{k}, n\right)\right] d \alpha,
$$

where

$$
\beta \Delta U_{i n s}(\mathbf{r}, n)=\beta \Delta U(\mathbf{r}, n)-\frac{K_{\alpha}}{2}\left(n-n_{\alpha}\right)^{2} .
$$

Now, it is possible to obtain an uniformly distributed sampling on the new variable $\alpha$. For appropriate values of $K_{\alpha}$ [i.e., $K_{\alpha} \simeq K$ and $n_{\alpha} \simeq n(\rho)$ ], we will have $\alpha_{\text {min }} \simeq-1$ and $\alpha_{1} \simeq 1$. Therefore,

$$
X_{s}=\frac{1}{N_{s} \Delta n} \sum_{k=1}^{N_{s}} \sqrt{\frac{2 \pi}{K_{\alpha}}}\left\langle\exp \left[-\beta \Delta U_{i n s}\left(\mathbf{r}_{k}, n_{k}\right)\right]\right\rangle_{\alpha} .
$$

When performing the insertion test using $\left\{\xi_{i}\right\}$ as internal coordinates, the intramolecular energy of the $N$ particles of the system does not change. In order to evaluate $\Delta U(\mathbf{r}, n)$ for a successful insertion, we have to compute: (i) the changes of the intermolecular interactions between the $N$ particles of the system $\Delta U_{\text {inter }, N}$ (since the values of $n_{i}$ will change for each particle whose $\rho_{i}$ is affected by the insertion), (ii) the intramolecular energy of the inserted particle, and (iii) the intermolecular interactions between the inserted particle and the particles of the system. The actual changes in $n\left(\rho_{i}\right)$ are small from the point of view of the intermolecular interactions and a full recalculation of all the pair interac- 
tions is not required. For a given successful insertion, the change in the potential index $\delta n_{i}$ is given by

$$
\delta n_{i}=n\left(\rho_{i, t e s t}\right)-n\left(\rho_{i}\right),
$$

where $\rho_{i, t e s t}$ corresponds to the equivalent bulk density of particle $i$ in the system with $N+1$ particles. The change of the intermolecular interactions between the $N$ particles controlled by the potential index $n_{i}, \delta u_{i}$ will be to a very good approximation

$$
\delta u_{i}=u_{i}^{\prime} \delta n_{i}+\frac{u_{i}^{\prime \prime}}{2}\left(\delta n_{i}\right)^{2}
$$

with

$$
\begin{gathered}
u_{i}=\frac{1}{2} \sum_{j \neq i} V\left(r_{i j}, n_{i}\right) \quad\left(r_{i j} \geqslant \sigma\right), \\
\Delta U_{\text {inter }, N}=\sum_{i=1}^{N}\left[u_{i}^{\prime} \delta n_{i}+\frac{u_{i}^{\prime \prime}}{2}\left(\delta n_{i}\right)^{2}\right],
\end{gathered}
$$

where $u_{i}^{\prime}$ and $u_{i}^{\prime \prime}$ are the first and second derivative of $u_{i}$ with respect to $n_{i}$ in the $N$-particle system. The derivatives are computed for each particle before performing a number of insertion attempts.

The evaluation of $\Delta U_{\text {intra }, N+1}$ is straightforward after evaluating the local density around the test particle.

The algorithm to perform the insertion test can be sketched according to the following steps: (i) Evaluate the local density around the test particle and determine the equivalent bulk density $\rho_{N+1}$. (ii) Compute the changes of the local densities of the $N$ particles of the system due to the insertion of the test particle and the values $\rho_{i, t e s t}$. (iii) Compute the changes in the intermolecular energy terms ascribed to the particles of the system, $\Delta U_{\text {inter }, N}$, and the interactions of these particles with the test particle in terms of the new potential indices $n_{i}$ of the $N$-particle system. (iv) Evaluate the intermolecular interaction of the test particle (and first and second derivatives with respect to $n_{N+1}$ ) at $n_{N+1}$ $=n\left(\rho_{N+1}\right)$. (v) Determine $K_{\alpha}$ and $n_{\alpha}$ as in internal moves and use them to pick a value of $n_{N+1}$. (vi) Compute the intramolecular energy of the test particle and the intermolecular interactions that depend on $n_{N+1}$ to finally calculate $\beta \Delta U_{\text {ins }}$. According to this scheme and the condition of equivalence to systems with global density interactions in the homogeneous limit, the configurational part of the chemical potential can be computed as

$$
\rho \sigma^{3} e^{-\beta\left(\mu-\mu^{0}\right)}=\left\langle\sqrt{\frac{K}{K_{\alpha}}} \exp \left[-\beta \Delta U_{i n s}\right]\right\rangle_{\alpha} .
$$

\section{RESULTS AND DISCUSSION}

Figure 2 shows the theoretical predictions for the phase diagram obtained for the full potential [Eqs. (1 and 2)] for $\alpha=12.0 v_{H S}$ (where $v_{H S}=\pi \sigma^{3} / 6$ ) and $n(0)=3.5$. The results obtained from TPT are in agreement with the RHNC predictions. Thus, we have relied on the TPT to search in the

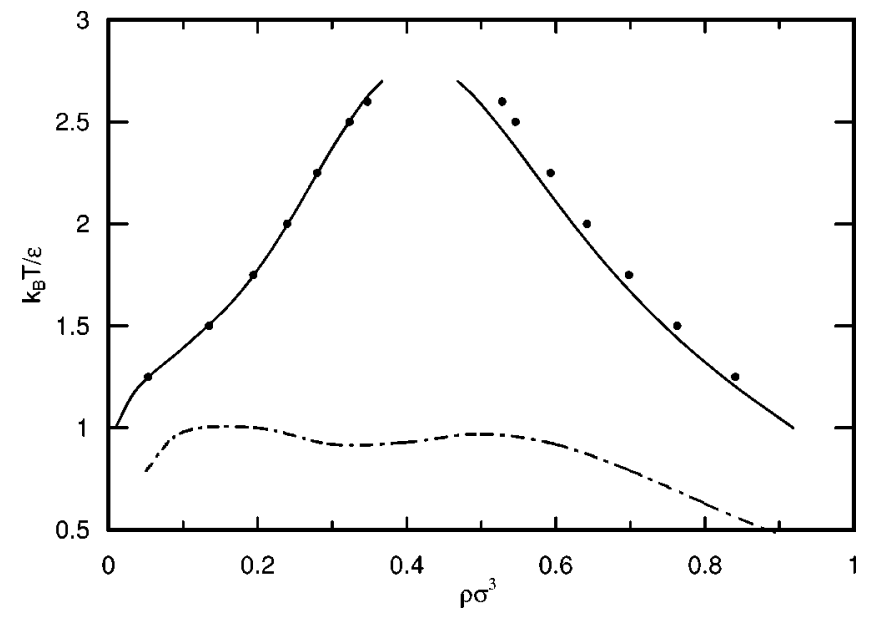

FIG. 2. Phase equilibrium in the density-temperature plane for the same parameters as in Fig. 1. The solid circles correspond to RHNC calculations and the solid lines are the results of first-order TPT. The no solution region of the RHNC equation is delimited by a dot-dashed line.

parameter space $(\alpha, n(0))$ in our quest for the liquid-liquid equilibrium. The good performance of the TPT is not surprising since the values of the potential index $n(\rho)$ for the cases considered correspond to a relatively slow decay of the attractions between particles. In Figs. 3 and 4 the TPT phase diagram is shown for different values of $\alpha$ and $n(0)$.

Finally, for $\alpha=5.7 v_{H S}, n(0)=3.2$, and $r_{c}=5.1 \sigma$ (other parameter combinations giving analogous qualitative results), it is seen in Fig. 5 that at low temperatures, the isotherms develop two van der Waals loops. The corresponding phase diagram, shown in Fig. 6, therefore, exhibits a vaporliquid transition and a liquid-liquid transition. Comparing these TPT estimates with the RHNC predictions, we find that even though the RHNC liquid-liquid equilibrium agrees with TPT, a pseudospinodal line with a single maximum covers the low-density region and prevents the determination of the vapor-liquid equilibrium. This situation is quite opposite to

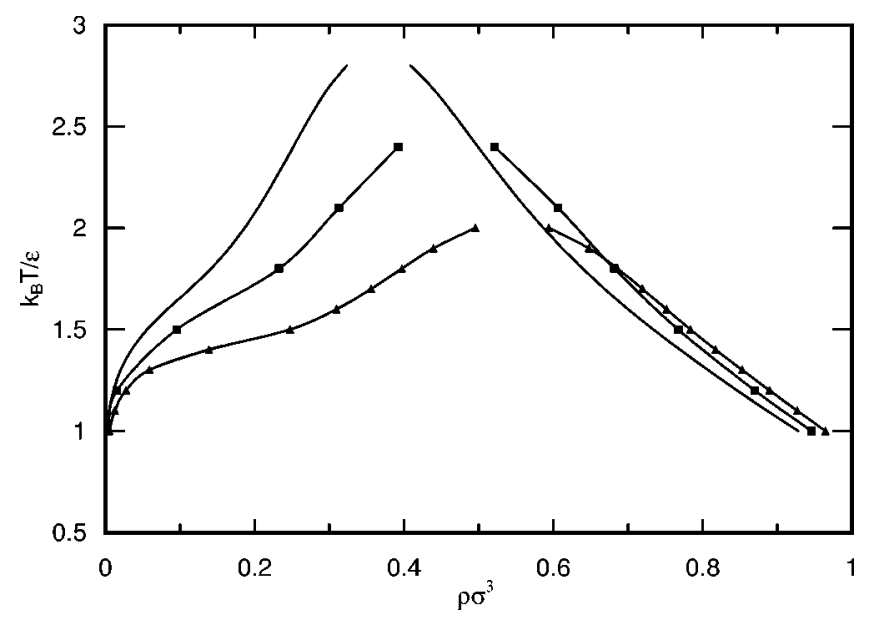

FIG. 3. Phase equilibrium in the density-temperature plane for $n(0)=3.2, r_{c}=5.1 \sigma$, and $\alpha=13 v_{H S}$ (solid line), $10 v_{H S}$ (solid line with squares), and $8 v_{H S}$ (solid line with triangles). 


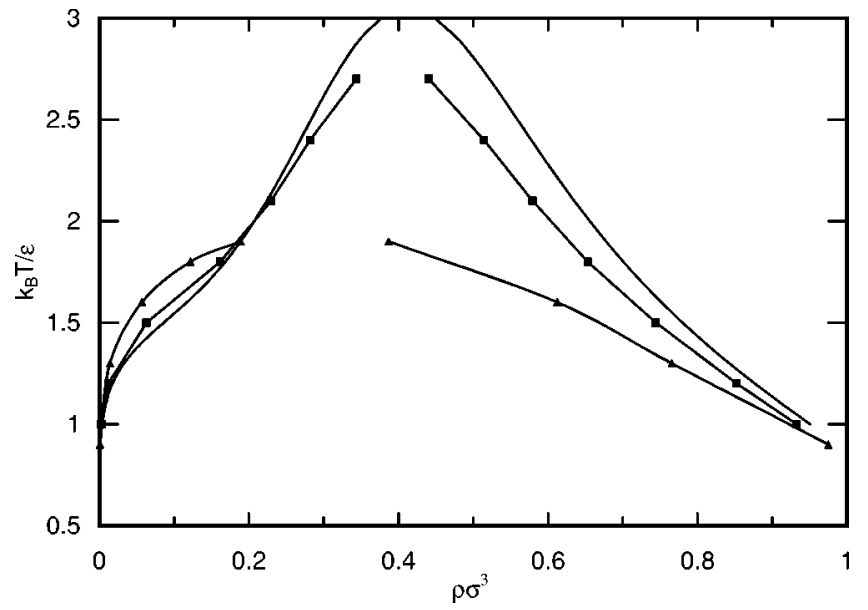

FIG. 4. Phase equilibrium in the density-temperature plane for $\alpha=12 v_{H S}, r_{c}=5.1 \sigma$, and $n(0)=3.4$ (solid line), 3.2 (solid line with squares), and 3.0 (solid line with triangles).

what was found in Fig. 2, where the divergence of correlations occurs well outside the binodal (an extreme case would be that of Ref. [10], where there is no divergence of correlations).

In order to get a rough estimate of the metastability limits (i.e., the binodal line for a global density simulation procedure) for fluids simulated in the $N V T$ ensemble, we have performed a series of simulations for different temperatures and densities with fixed potential indices and $r_{c}=5.1 \sigma$. From the chemical potentials calculated using simulations with $N=256$ the liquid-vapor equilibrium (LVE) can be determined. The values of the inverse reduced temperature $1 / t$ $=\epsilon /\left(k_{B} T\right)$ and the potential index considered lie in the ranges $[0.7,1.0]$ and $[3.2,3.6]$, respectively. The results of the LVE for fluids with density-independent interactions can be fitted to the following equations:

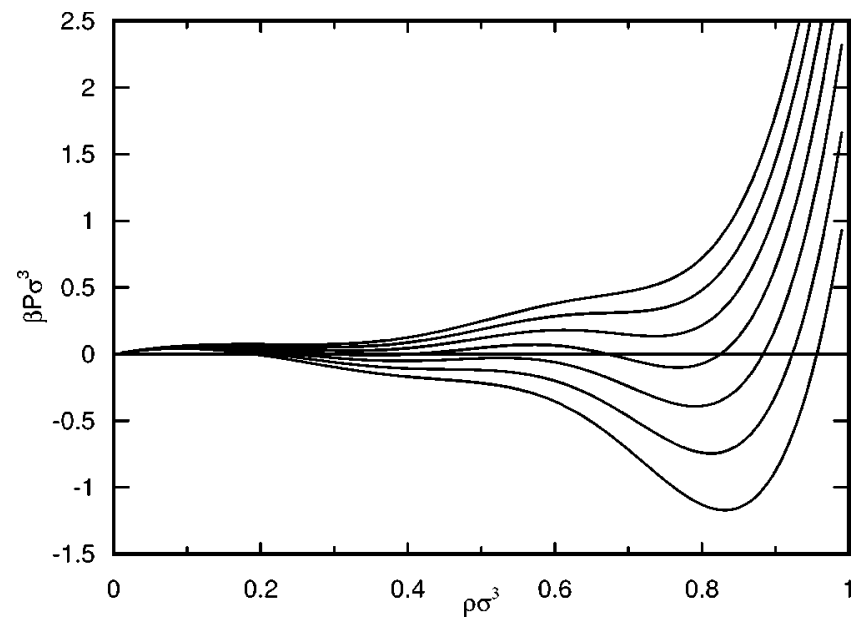

FIG. 5. Isotherms in the density-pressure $\left(p^{*}=\beta p \sigma^{3}\right)$ plane obtained from first-order TPT for $\alpha=5.7 v_{H S}, n(0)=3.2$, and $r_{c}$ $=5.1 \sigma$ and reduced temperatures (from bottom to top) $t=0.9$ -1.2 by steps of 0.05 . It is seen that at low temperatures the isotherms exhibit two van der Waals loops.

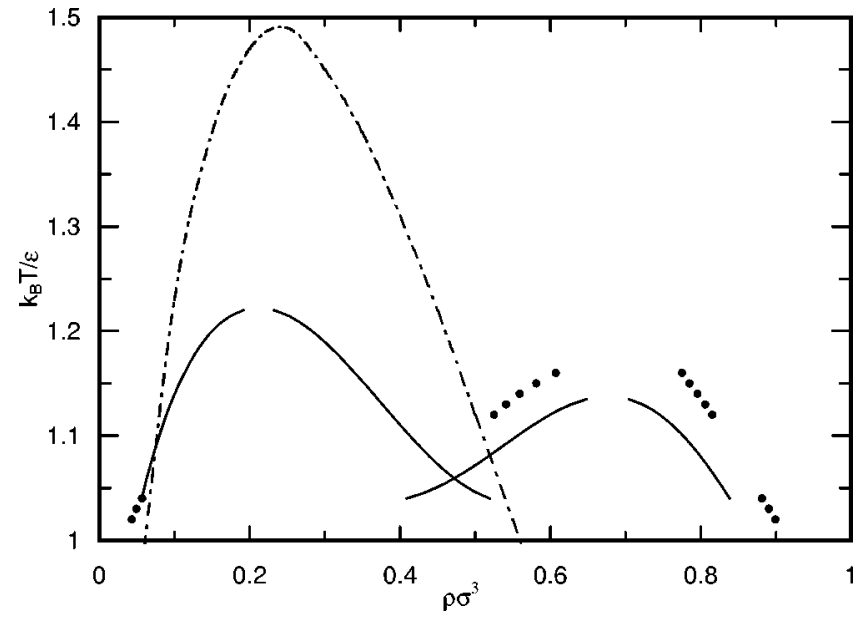

FIG. 6. Phase equilibrium from first-order TPT (solid lines) and RHNC (solid circles) for $\alpha=5.7 v_{H S}, n(0)=3.2$, and $r_{c}=5.1 \sigma$. The no solution region of the RHNC equation is delimited by a dotted line.

$$
\begin{gathered}
\frac{\rho_{l} \sigma^{3}+\rho_{v} \sigma^{3}}{2}=b_{0}+b_{t} \frac{1}{t}+b_{n} n, \\
\rho_{l} \sigma^{3}-\rho_{v} \sigma^{3}=\left[a_{0}+a_{t} \frac{1}{t}+a_{n} n\right]^{1 / \beta^{\prime}},
\end{gathered}
$$

where $\rho_{l}$ and $\rho_{v}$ are the densities at coexistence of the liquid and the vapor phases, and the exponent $\beta^{\prime}$ has been considered as an adjustable parameter. A weighted least squares fit of the data yields: $b_{0}=0.420764, b_{t}=0.329877, b_{n}=$ $-0.104065, \quad \beta^{\prime}=0.3842, \quad a_{0}=0.872437, a_{t}=1.728806$, and $a_{n}=-0.618653$.

The metastability limits for a fluid with the densitydependent pair potential (3) for $\alpha=5.7 v_{H S}, n(0)=3.2$, and $r_{c}=5.1 \sigma$, were obtained as a function of the density by searching for the value of $T$, where either $\rho=\rho_{v}(T, n(\rho))$ or $\rho=\rho_{l}(T, n(\rho))$. The results are shown in Fig. 7 (dotted line). It is remarkable the agreement between the binodal lines estimated using global density MC results and the pseudospinodal lines arising from RHNC calculations. Comparing Figs. 6 and 7 it seems that the fluid's stability below the no solution line of the RHNC and above the vapor-liquid critical point stems from the density dependence of the interactions. Simulations using the local density procedure were carried out in the NVT ensemble for the same set of parameters. The number of particles were chosen to be $N=500$ in most of the cases. Some simulations were run using $N=864$ and $N$ $=1372$ in order to analyze the size effects.

For a given temperature, we usually performed 24 runs at packing fractions $\eta=\rho \pi \sigma^{3} / 6$ given by $\eta=0.02 i \quad(i$ $=1,2, \ldots, 24)$. Initial configurations were taken either from a lattice structure or from the last configuration of a run at higher density. Simulations were organized in cycles, each cycle implying $N$ translational attempts and $N$ internal moves. For each case, we ran $4 \times 10^{4}$ cycles and averages were taken over the second half of the runs. After completing 


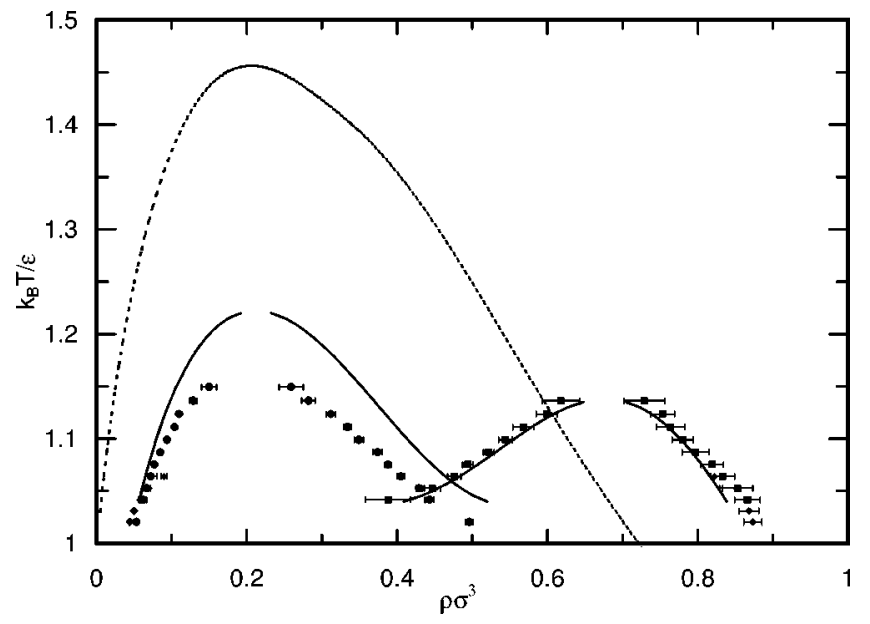

FIG. 7. Phase equilibria obtained from the local density simulation technique (solid circles) for $\alpha=5.7 v_{H S}, n(0)=3.2$, and $r_{c}$ $=5.1 \sigma$, compared with first-order TPT (solid curves). The region where we ought to use the local density criteria in the simulation technique is also indicated (dotted line).

each cycle, we performed typically $10^{3}$ insertion attempts to evaluate the chemical potential. At a given temperature, the chemical potential estimates were fitted as a function of the density using Eq. (24). From the coefficients $a_{k}$ the pressure and the phase equilibrium were determined.

In Table III the coexisting densities and the pressure at coexistence for the vapor-liquid and the liquid-liquid equilibria are gathered, whereas in Table IV the vapor-high density liquid equilibrium data are reported. The system size dependence of the results is only significant (within error bars) close to the critical points. In Fig. 7 the phase diagram obtained from simulation results and TPT is shown. The good
TABLE IV. Simulation results for the reduced coexisting densities, pressure and fugacity $z$ of the vapor $(v)$-high density liquid $(h l)$ equilibrium for the same parameters and inverse reduced temperatures as defined in Table III.

\begin{tabular}{lccccl}
\hline \hline$t^{-1}$ & $N$ & $\rho_{v}^{*}$ & $\rho_{h l}^{*}$ & $p_{v-h l}^{*}$ & \multicolumn{1}{c}{$\ln z$} \\
\hline 0.94 & 500 & $0.090(4)$ & $0.823(19)$ & $0.0489(5)$ & $-3.348(11)$ \\
0.94 & 864 & $0.089(3)$ & $0.822(16)$ & $0.0486(4)$ & $-3.351(5)$ \\
0.95 & 500 & $0.070(2)$ & $0.851(22)$ & $0.0439(5)$ & $-3.419(7)$ \\
0.96 & 500 & $0.058(2)$ & $0.868(17)$ & $0.0394(7)$ & $-3.495(17)$ \\
0.97 & 500 & $0.0498(9)$ & $0.868(13)$ & $0.0358(4)$ & $-3.568(8)$ \\
0.98 & 500 & $0.0443(8)$ & $0.873(12)$ & $0.0330(4)$ & $-3.632(9)$ \\
\hline \hline
\end{tabular}

agreement between theory and simulation, specially in the liquid-liquid equilibrium is due to the relatively slow decay of the pair potential.

In the simulation method proposed here, we have included a new length scale in the system, basically controlled by the parameter $\lambda$. In order to get a precise analysis of the fluid phase equilibria, especially close to the critical points, without significant effects on the particular choice of the local density parameters, it would be required that $\sigma \ll \lambda \sigma$ $\ll L$. In situations where the fluid-fluid equilibrium is produced by the density dependence of the pair potential, the local density approach could be expected to induce a crossover (basically controlled by the length $\lambda \sigma$ ) from a meanfield critical behavior (where no local density considerations are taken into account, $\lambda \rightarrow \infty)$ to an Ising-like behavior provided that the interactions are short ranged [20].

Finally, the strategy followed in the simulation of a fluid with local density dependence of the potential (i.e., the introduction of "fictitious" internal degrees of freedom) could be

TABLE III. Reduced densities $\rho^{*}=\rho \sigma^{3}$ and pressure $p^{*}=\beta p \sigma^{3}$ at coexistence obtained from Monte Carlo simulation using the local density method, for $n(0)=3.2, \alpha=5.7 v_{H S}, r_{c}=5.1 \sigma, \lambda=4$, and $q=6$, at different reduced inverse temperatures $t^{-1}=\epsilon / k_{B} T$ and number of particles $N$. Columns 3 to 5 correspond to the vapor-liquid coexistence, and columns 6 to 8 to the liquid-liquid coexistence. The subindices $v, l l$, and $h l$ refer to the vapor, the low density liquid, and the high density liquid, respectively. In the parentheses, we report the estimation of error bars as twice the standard deviation of the mean, in units of the last figure of the corresponding magnitude.

\begin{tabular}{|c|c|c|c|c|c|c|c|}
\hline$t^{-1}$ & $N$ & $\rho_{v}^{*}$ & $\rho_{l l}^{*}$ & $p_{v-l l}^{*}$ & $\rho_{l l}^{*}$ & $\rho_{h l}^{*}$ & $p_{l l-h l}^{*}$ \\
\hline 0.87 & 500 & $0.150(10)$ & $0.259(16)$ & $0.0620(3)$ & & & \\
\hline 0.88 & 500 & $0.124(3)$ & $0.294(6)$ & $0.0591(2)$ & $0.618(25)$ & $0.729(27)$ & $0.286(7)$ \\
\hline 0.88 & 864 & $0.129(5)$ & $0.282(9)$ & $0.0593(3)$ & $0.638(41)$ & $0.704(46)$ & $0.290(6)$ \\
\hline 0.89 & 500 & $0.110(2)$ & $0.312(6)$ & $0.0564(3)$ & $0.599(14)$ & $0.753(16)$ & $0.257(6)$ \\
\hline 0.89 & 864 & $0.113(2)$ & $0.315(5)$ & $0.0566(2)$ & $0.598(14)$ & $0.745(19)$ & $0.255(5)$ \\
\hline 0.90 & 500 & $0.101(2)$ & $0.332(6)$ & $0.0541(2)$ & $0.570(12)$ & $0.764(19)$ & $0.219(5)$ \\
\hline 0.90 & 864 & $0.104(2)$ & $0.334(5)$ & $0.0543(2)$ & $0.568(14)$ & $0.758(26)$ & $0.217(6)$ \\
\hline 0.90 & 1372 & $0.104(2)$ & $0.332(5)$ & $0.0543(2)$ & $0.566(9)$ & $0.756(17)$ & $0.219(4)$ \\
\hline 0.91 & 500 & $0.094(2)$ & $0.349(6)$ & $0.0519(3)$ & $0.544(9)$ & $0.780(14)$ & $0.183(6)$ \\
\hline 0.92 & 500 & $0.085(1)$ & $0.374(6)$ & $0.0495(2)$ & $0.522(7)$ & $0.797(18)$ & $0.152(5)$ \\
\hline 0.93 & 500 & $0.077(2)$ & $0.388(4)$ & $0.0469(2)$ & $0.494(7)$ & $0.819(15)$ & $0.115(5)$ \\
\hline 0.94 & 500 & $0.0725(8)$ & $0.405(5)$ & $0.0452(2)$ & $0.476(9)$ & $0.833(16)$ & $0.084(6)$ \\
\hline 0.94 & 864 & $0.0729(8)$ & $0.407(5)$ & $0.0452(2)$ & $0.472(8)$ & $0.830(13)$ & $0.081(5)$ \\
\hline 0.95 & 500 & $0.0669(5)$ & $0.429(4)$ & $0.0431(2)$ & $0.447(11)$ & $0.853(20)$ & $0.053(7)$ \\
\hline 0.96 & 500 & $0.0620(5)$ & $0.443(6)$ & $0.0410(22)$ & $0.388(30)$ & $0.866(17)$ & $0.021(6)$ \\
\hline 0.98 & 500 & $0.0533(4)$ & $0.496(5)$ & $0.0371(2)$ & & & \\
\hline
\end{tabular}


applied to other situations where many body effects have to be considered. A good example is the simulation of models in which the polarizability is explicitly taken into account [21]. Following such a route one can avoid these problems with the evaluation of the energy using iterative procedures $[22,23]$.

\section{ACKNOWLEDGMENTS}

The authors acknowledge financial support from the Dirección General de Enseñanza Superior e Investigación Científica (DGESCYT) under Grant Nos. PB 98-0673-C02-02 and BFM-2001-1017-C03 (E.L., G.R., and C.F.T.)
[1] C.N. Likos, Phys. Rep. 348, 267 (2001).

[2] J. Hafner, From Hamiltonians to Phase Diagrams (Springer, Berlin, 1985).

[3] B.V. Derjaguin and L.D. Landau, Appl. Sci. Res., Sect. B 14, 633 (1941).

[4] E.J.W. Verwey and J.T.G. Overbeek, Theory of the Stability of Lyophobic Colloids (Elsevier, Amsterdam, 1948).

[5] B. Smit, T. Hauschild, and J.M. Prausnitz, Mol. Phys. 77, 1021 (1992).

[6] F.H. Stillinger, H. Sakai, and S. Torquato, J. Chem. Phys. 117, 288 (2002).

[7] P.H. Poole, T. Grande, C.A. Angell, and P.F. McMillan, Science 275, 322 (1997); S. Harrington, R. Zhang, P.H. Poole, F. Sciortino, and H.E. Stanley, Phys. Rev. Lett. 78, 2409 (1997).

[8] C.F. Tejero and M. Baus, Phys. Rev. E 57, 4821 (1998).

[9] N.G. Almarza, E. Lomba, G. Ruiz, and C.F. Tejero, Phys. Rev. Lett. 86, 2038 (2001).

[10] M. Dijkstra and R. van Roij, J. Phys.: Condens. Matter 10, 1219 (1998).

[11] A.A. Louis, J. Phys.: Condens. Matter 14, 9187 (2002).

[12] However, it is likely that free-energy barriers will produce an increase of hysteresis in phase transitions when working with $N p T$ or $\mu V T$ ensembles.

[13] D. Frenkel and B. Smit, Understanding Molecular Simulation (Academic Press, San Diego, 1996).

[14] F. Lado, S.M. Foiles, and N.W. Ashcroft, Phys. Rev. A 28, 2374 (1983).

[15] A.G. Schlijper, M.M. Telo da Gama, and P.G. Ferreira, J. Chem. Phys. 98, 1534 (1993).

[16] C.F. Tejero and M. Baus, J. Chem. Phys. 118, 892 (2003).

[17] C.F. Tejero, J. Phys. Condens. Matter 15, 5395 (2003).

[18] J.P. Hansen and I.R. McDonald, Theory of Simple Liquids (Academic, Oxford, 1986).

[19] M.P. Allen and D.J. Tildesley, Computer Simulation of Liquids (Clarendon, Oxford, 1987).

[20] P.J. Camp and G.N. Patey, J. Chem. Phys. 114, 399 (2001).

[21] K. Kiyohara, K.E. Gubbins, and A.Z. Panagiotopoulos, J. Chem. Phys. 106, 3338 (1997).

[22] M.G. Martin, B. Chen, and J.I. Siepmann, J. Chem. Phys. 108, 3383 (1998).

[23] D. Frenkel and B. Smit, Understanding Molecular Simulation (Academic, San Diego, 1996). 